\title{
Assessing Upper-extremity Motion: An Innovative, Objective Method to Identify Frailty in Older Bedbound Trauma Patients
}

Nima Toosizadeh ${ }^{1,2}$, Bellal Joseph ${ }^{3}$, Michelle R. Heusser ${ }^{1,4,5}$, Tahereh Orouji Jokar $^{3}$, Jane Mohler ${ }^{1,2,5}$, Herb A. Phelan ${ }^{6}$, Bijan Najafi $1,2,5,7$

1: Interdisciplinary Consortium on Advanced Motion Performance (iCAMP), Department of Surgery, University of Arizona, Tucson, Arizona, USA

2: Arizona Center on Aging, Department of Medicine, University of Arizona, Tucson, AZ, USA

3: Division of Trauma, Emergency Surgery, Critical Care, and Burns, Department of Surgery, University of Arizona, Tucson, AZ, USA

4: Department of Bioengineering, University of Pittsburgh, Pittsburgh, PA, USA

5: Department of Biomedical Engineering, University of Arizona, Tucson, Arizona, USA

6: Division of Burns, Trauma, and Critical Care, Department of Surgery, UT-Southwestern Medical Center, Dallas, Texas, USA

7: Interdisciplinary Consortium on Advanced Motion Performance (iCAMP), Michael E. DeBakey Department of Surgery, Baylor College of Medicine, Houston, Texas, USA

\section{Corresponding author:}

Bijan Najafi, PhD

Michael E. DeBakey Department of Surgery

Baylor College of Medicine

One Baylor Plaza, MS: BCM390

Houston, TX 77030

Phone: (713) 798- 7536

E-mail: najafi.bijan@gmail.com

Key words: upper-limb movement, emergency, frailty, hospitalization, urgent care, fall incident. 
Previous Presentations: Two abstracts based on part of results of this study were presented at Biomedical Engineering Society (BMES), San Antonio, Texas \& Gerontology Society of America (GSA), Washington, DC with following citations:

1. Heusser M., Toosizadeh N., Zangbar-Sabegh B., Mohler J., Joseph B., Najafi B., (2014) Upper Extremity Frailty Assessment in Trauma Patients Using Wearable Sensor Technology. Biomedical Engineering Society (BMES), San Antonio, Texas.

2. Toosizadeh N., Heusser M., Zangbar-Sabegh B., Joseph B., Mohler J., Najafi B. (2014) Objective Frailty Assessment in Trauma Patients using a Novel Upper-extremity Approach. Gerontology Society of America (GSA), Washington, DC. 


\section{Abstract:}

Background: Despite increasing evidence that assessing frailty facilitates medical decisionmaking, a quick and clinically simple frailty assessment tool is not available for trauma settings. Study Design: This study examined accuracy and acceptability of a novel wearable technology (upper-extremity frailty: UEF) to objectively assess frailty status in older adults ( $\geq 65$ years) admitted to the hospital due to traumatic ground-level falls. Frailty was measured using a validated modified Rockwood questionnaire, the Trauma-Specific Frailty Index (TSFI), as the gold standard. Participants performed a $\sim 20$-second trial of rapid elbow flexion with the dominant elbow in a supine posture while wearing the UEF system. Results: We recruited 101 eligible older adults (Age: $79 \pm 9$ years). UEF parameters indicative of slowness, weakness, and exhaustion during elbow flexion were independent predictors of the TSFI score, while adjusted for age, gender, and body mass index. A high agreement $(r=0.72, p<.0001)$ was observed between TSFI score and UEF model; sensitivity and specificity for predicting the frailty status were $78 \%$ and $82 \%$, respectively. Of recruited participants $57 \%$ were not able to walk at the time of measurements, suggesting a limitation for walking-based frailty assessments. Significant correlations were observed between UFE parameters and number of falls within a prior year, with highest correlation observed for elbow flexion slowness $(r=-0.41)$. Conclusion: The results suggest that a simple test of 20 -second elbow flexion is practical and sensitive to identify frailty among hospitalized older adults. The UEF test is independent of walking assessments, reflects several frailty markers, and it is practical for bedbound patients. 


\section{Introduction}

The population of older adults is rapidly growing; those over the age of 65 now account for $13 \%$ of the population in the United States, and this percentage is expected to increase to 20\% (72.1 million) by 2030 (1). Among older adults, falls are the most common mechanism of traumatic injury, with over two million fall-related emergency department visits each year (2). Although age is strongly associated with adverse health outcomes due to traumatic geriatric injuries, the response of an older adult to traumatic events is highly variable due to the heterogeneity of aging (3). The concept of "frailty" is useful in identifying homeostenotic older adults with low physiological reserve and high vulnerability to poor outcomes including longer length of hospital stay, higher rate of surgical complications, and increased risk of disability, institutionalization, and death $(4,5)$. Previous studies have demonstrated that frailty is a more sensitive predictor of adverse health outcomes than is chronological age in medical and surgical patients $(6,7)$. Frail patients are 2.5 times more likely to have a longer length of hospital stay, and are 20 times as likely to be discharged to a nursing home or assisted living facility, than are non-frail individuals (7).

We previously developed and validated (using the Fried index (4)) an upper-extremity frailty (UEF) assessment method among community dwelling older adults (8). The UEF assessment method integrates low cost sensors and the physical task is easily performed in under one minute. The uniqueness of the proposed technology, unlike the Fried index that requires a gait assessment, is in its applicability in older non-ambulatory patients, such as those in trauma settings. Additionally, the UEF method provides an objective way to measure frailty status because it does not rely on subjective questionnaires, but rather it includes objective motion parameters and demographic information. 
The purpose of the current study was to validate the accuracy, and to explore the acceptability and feasibility of the UEF assessments in older adults who were admitted to the hospital due to traumatic ground-level falls. We hypothesized that: 1) UEF assessments would accurately categorize participants into frail and non-frail groups (compared to a modified Rockwood frailty score as the gold standard); and 2) a larger percentage of participants would be able to perform the UEF assessments compared to the Fried index (which requires a walking speed

measurement). As a secondary aim we explored the association between UEF parameters and the number of falls within one year prior to hospitalization in this sample of high fall risk older adults. In the previous study we determined strong correlations between upper-extremity motion and gait speed (8), and since gait speed and fall risk are associated $(9,10)$, we, therefore, hypothesized significant associations would exist between UEF parameters and the number of retrospective falls.

\section{Methods}

\section{Participants}

A sample of 101 older adult patients with ground-level fall injuries was recruited from the Division of Trauma, Critical Care, and Emergency Surgery service at the University of Arizona. Inclusion criteria were: 1) aged 65 years and older, 2) ability to understand study instructions, and 3) having had at least one ground-level fall causing an injury within the prior two weeks that led to hospital admission. Participants were excluded if they had significant upper-extremity disorders in both arms (e.g., bilateral fractures or rheumatoid arthritis with elbow or shoulder involvement), or if they were in a physical condition (e.g., such as severe head injury and unconsciousness) that made them unable to perform the UEF test. The study was approved by the University of Arizona Institutional Review Board. Before participation, written informed consent was obtained by trained research coordinators according to the principles expressed in 
the Declaration of Helsinki (11) from all participants, or from an authorized person if a participant was clinically assessed as lacking capacity for informed consent.

\section{Frailty assessment}

Frailty was measured using a validated modified Rockwood questionnaire, the Trauma-Specific Frailty Index (TSFI) (12), which is based on 15 trauma-specific variables. Using TSFI, participants were divided into non-frail (score $\leq 0.27$ ) and frail (score $>0.27$ ) groups. The TSFI method has been validated for predicting unfavorable discharge disposition (i.e., discharge to skilled nursing facility or rehabilitation center instead of home) and death (12). Number of falls within the prior year of the measurement was recorded as a part of TSFI.

\section{UEF assessment and acceptability measures}

Each participant performed a $\sim 20$-second trial of elbow flexion, within which they repetitively flexed and extended their dominant elbow to full flexion and extension as quickly as possible in supine posture (bedbound position) while wearing the UEF system. Participants performed the elbow flexion task with their non-dominant side in case of injury or attachments of vital sign monitoring equipment. Before the actual test, participants performed a short practice trial to become familiar with the protocol. The protocol was explained to participants and they were encouraged only once, before elbow flexion, to do the task as fast as possible (participants were not further encouraged during the task). Wearable sensor technology was used to measure forearm and upper-arm motion. A triaxial wearable gyroscope sensor (sample frequency $100 \mathrm{~Hz}$, BioSensics LLC, Cambridge, MA) was attached to the upper-arm near the biceps and one to the wrist using a band attached with Velcro, to estimate three-dimensional angular velocity of the upper-arm and forearm segments, and ultimately the elbow angular velocity. 
Several outcome measures representing kinematics and kinetics of elbow flexion were derived using angular velocity and anthropometric data (i.e., participants' stature and body mass).

Outcome measures included: 1) speed; 2) flexibility; 3) power; 4) rise time; 5) moment; 6) speed variability; 7) speed reduction; and 8) flexion number (see Table 1 for definitions). These parameters were defined to quantify "slowness", "weakness", and "exhaustion" as Fried criteria (4), as well as "flexibility" as an additional frailty marker (13). Slowness was assessed by measuring speed, rise time, and flexion number; weakness was assessed by measuring power and moment; exhaustion was assessed by speed variability and speed reduction. (Readers are referred to (8) for more details regarding validation of UEF using a motion capture system, and for a detailed description of parameter calculations.)

To assess the acceptability of the UEF test compared to the Fried index among hospitalized patients, their ability to walk was determined by either participants' verbal confirmation or examiner judgement. Further, participants' ability to independently complete the TSFI test was assessed, as several participants could not effectively address all components of the TSFI test due to injury or cognitive impairments.

\section{Statistical analysis}

UEF parameters were compared between two frailty groups (i.e., non-frail and frail based on TSFI) using separate analyses of variance (ANOVAs) with age, gender, and body mass index (BMI) as covariates. ANOVAs and $\chi^{2}$ tests were performed to evaluate the differences in demographic parameters between two groups.

Further, a set of data from the first 35 non-frail and 35 frail participants (training dataset) was used to develop the UEF multivariate regression model for predicting the frailty score. In this model, independent associations between UEF parameters and frailty were assessed, 
considering frailty score as the dependent variable, UEF parameters as independent variables, and age, gender, and BMI as covariates. Only UEF parameters with a significant association $(p<.05)$ with frailty from ANOVAs were entered into the model. A set of 31 subsequent participants (validation dataset) was then used to assess the accuracy of the UEF model in predicting the frailty score and the frailty status in comparison with TSFI, using UEF parameters that demonstrated significant independent association with TSFI within the training database. For evaluating the model, correlations between model-predicted and TSFI frailty scores were assessed using linear ANOVA and reported as Pearson correlations $(r)$. The goodness of fit was examined by testing for the normality of the residuals (Shapiro-Wilk $W$-test) and comparing the predicted and measured frailty scores using paired $t$-test. Further, frailty status prediction using the UEF model was assessed by comparison to the TSFI frailty status, and sensitivity and specificity were calculated.

To assess the association between the UEF test and fall risk, correlations between UEF parameters (i.e., speed, flexibility, power, rise time, moment, speed variability, speed reduction, and flexion number) and number of falls in the preceding year (recorded within TSFI) were assessed using linear Pearson correlations for normally distributed or the Spearman's rank for non-normally distributed samples (both reported as $r$ values). Cut-offs for correlations were selected as follows: 0.01-0.19 were negligible; 0.20-0.29 were weak; 0.30-0.39 were moderate; 0.40-0.69 were strong; and 0.70-1.00 were very strong (14). All analyses were performed using JMPTM (version 11; SAS Institute Inc., Cary, N.C., USA), with a significance level of $p<.05$. Summary results are presented as means with standard deviation (SD) and standard errors (SE).

\section{Results}




\section{Participants}

One hundred fifty-five older adults who satisfied inclusion criteria were screened for the purpose of this study. Fifty-four (35\%) were excluded since they were unable to perform UEF measurement, mainly because of unconsciousness or severe injuries due to fall. Thus, 101 participants were consented for the purpose of this study. Among those recruited, $32 \%$ were not able to perform the UEF with the dominant arm; thus, the data from non-dominant arm was captured. Moreover, $7 \%$ of the recruited participants were not able to complete the TSFI test independently, mainly due to inability to provide correct answers due to injury, memory problems, or emotional lability upon questioning. For these participants, a relative or caregiver assisted in completion of the TSFI test. Among recruited participants $57 \%$ were not able to walk at the time of the assessment, and, therefore, would have not been able to perform walking related frailty assessment tools such as the Fried index.

Among 101 recruited participants, 52 (51\%) were non-frail based on TSFI, with mean (SD) age and BMI of $78(10)$ years and $25.30(4.46) \mathrm{kg} / \mathrm{m}^{2}$, respectively. Corresponding values were 80 (9) years and $24.93(4.85) \mathrm{kg} / \mathrm{m}^{2}$ for frail participants. Age was not significantly different between non-frail and frail participants. The number of falls within one year prior to measurements was $\sim 5$ times higher among frail participants $(p<.0001)$. Other sociodemographic information is reported in Table 2.

\section{Association between the UEF test and TSFI}

From ANOVAs, all parameters extracted from the UEF test, except speed variability, were significantly different between non-frail and frail groups (Table 2). The highest effect sizes were observed for slowness as quantified by speed of arm movement $(d=1.50)$, number of flexions during the 20 -second test $(d=1.18)$, and weakness as quantified by elbow power and moment $(d=1.10)$. Speed of elbow flexion (a feature of slowness) was $45 \%$ less among frail when 
compared to the non-frail group $(p<.0001, d=1.50)$. On the same note, power of movement (another feature of weakness) was $74 \%$ less among frail compared to the non-frail group $(p<.0001, d=1.10)$. The results from elbow flexion showed that speed reduction (an indicator of exhaustion) was $72 \%$ greater in frail compared to the non-frail group $(p<.01, d=0.51)$. Furthermore, the number of flexions and the flexibility measure were $32 \%$ and $27 \%$ less, respectively, among frail participants when compared to the non-frail group $(p<.0001, d=1.18)$.

From logistic regression results using the training dataset, a Pearson correlation coefficient of $0.72(p<.0001)$ was achieved in predicting the TSFI score using the UEF model; sensitivity and specificity for predicting the frailty status were $94 \%$ and $86 \%$, respectively. Further, a $W$-test revealed normality of residuals $(W=0.98, p=0.23)$, and $t$-test results showed no significant difference between the UEF model score and the TSFI score $(p=0.99)$. Results from this regression model revealed that speed (slowness), power (weakness), and speed reduction (exhaustion) UEF parameters were independent predictors of the TSFI score (Table 3). Age, gender, and BMI were not significantly associated with the TSFI score when considered as independent variables in addition to UEF parameters in the model. Using the UEF model with speed, power, and exhaustion as independent variables, a Pearson correlation coefficient of $0.74(p<.0001)$ was obtained in predicting the frailty score in the subsequent validation dataset (Figure 1). (Of note, UEF parameters and frailty scores of these participants were not used to develop the UEF model). Sensitivity and specificity values of $78 \%$ and $82 \%$ were achieved for predicting the frailty status in the validation dataset; $t$-test showed no significant difference between UEF and TSFI scores $(p=0.79)$.

\section{Correlation between the UEF test and fall risk}

Except for speed variability, all UEF parameters were significantly correlated with the number of falls within one year prior to hospitalization. Among these, flexibility, moment, and speed 
reduction revealed weak ( $r=0.20-0.25)$, speed, power, and flexion number showed moderate $(r$ $=0.31-0.37)$, and rise time exhibited strong $(r=-0.41)$ correlations with the number of historical falls.

\section{Discussion}

UEF assessment in bedbound hospitalized patients

As hypothesized, it was possible to categorize frailty groups with high sensitivity and specificity using a quick, simple upper-extremity task. Although we previously validated this model in community dwelling older adults (8), the test was not assessed in bedbound and nonambulatory older adults. In addition, several additional factors such as injury severity or injured area (head versus body) might have influenced the accuracy of the UEF test in the traumarelated hospitalized patients, which were not addressed in the previous study. As current results suggest, measuring physical function at the maximum performance level (maximum flexion speed) during the hospitalization period correlated well with the overall frailty score based on the condition within two weeks prior to the fall. However, worse UEF performance was observed among hospitalized participants in the current study compared to non-hospitalized community dwelling participants from our previous study (8) when adjusted for age, gender, and BMl; specifically, speed, flexibility, and power were $30 \%$ less $(p<.001)$, and speed variability and speed reduction were $58 \%$ larger among trauma patients $(p<0.02)$. Of note, no adjustment of frailty status was done since different frailty assessment approaches were used in the two studies. Previous studies have also demonstrated deterioration in performing daily activities (15) and reduction in gait-dependent frailty performance (16) after hospitalization and intervening illness. Therefore, the observed differences in UEF performance due to hospitalization were expected. This observation should, however, be confirmed in a prospective experimental setting that measures alterations in UEF parameters pre- and post-hospitalization. Overall, our results suggest that although UEF performance may decline due to fall-related injuries and 
hospitalization-related deconditioning, the reduced performance is systematic and UEF performance correlates well with the subjective frailty score.

\section{Association between upper-extremity motion and fall risk}

Results demonstrated a strong association with UEF rise time $(r=-0.41)$ and the number of historical falls over the past year. These findings are in agreement with our previous results, where the strongest correlation $(r=0.68, p<.001)$ was observed between UEF rise time and gait speed (8). Fukagava et al. reported that older adults with a history of falling had less than half of the knee and ankle strength of non-fallers (17). In this study, a decreased lower-extremity muscle strength in individuals with a history of falling was correlated with a slower gait speed compared to non-fallers (17). In addition to associations between lower-extremity weakness and fall risk, previous work has also demonstrated that older adults with a history of falling have lower grip strength when compared to non-fallers (18).

\section{Limitations}

First, as with measurement limitations in gait-based frailty measures, upper-extremity disability or injury may limit measurements; we found this to be the case for some individuals who were excluded from the study because they were unable to perform the UEF task. That said, it is likely that this limitation would also apply to measuring grip strength, which would likewise limit measurements within the Fried index. Furthermore, due to upper-extremity attachment of vital sign monitoring equipment within the hospital setting, $32(32 \%)$ participants were not able to perform the UEF test on their dominant arm, thus the assessment was performed using the nondominant arm. However, this is likely to be less important, as our previous findings demonstrated similar prediction qualities for assessing frailty using either the dominant or the non-dominant arm (8). 
Second, several screened individuals (35\%) that were admitted to the hospital were not able to perform the UEF test due to severe head injuries or unconsciousness. Although several fall patients were not able to perform the UEF test, this limitation also co-exists for other common frailty measures such as Fried (gait-limited) or Rockwood (memory-limited). Further, although we limited our sample to those trauma patients with only ground-level falls, our statistical analyses were not adjusted for potentially confounding injury severity scores. In our investigation the UEF frailty score demonstrated strong association with frailty, regardless of severity of injury; thus, the UEF frailty score was not adjusted with any injury scores such as the Injury Severity Score (19).

\section{Clinical Relevance}

Older adults who experience ground-level falls and other geriatric trauma have wide heterogeneity; some may quickly heal, while others may experience permanent changes in their levels of function and independence. Goals of care must be delineated soon after hospital admission, supporting the clinical goals of wise resource allocation, and the provision of high quality, cost-effective geriatric trauma care. The UEF test has high potential for prognostic prediction to determine who will benefit from aggressive injury management versus those who may benefit from less aggressive palliative care. Future research to characterize outcomes of geriatric trauma care and to assist with determination of management strategies based on the UEF frailty status are needed. The proposed approach could be integrated in a precision medicine model to improve customization of care using individualized, objective, phenotypic data on function and overall health status (20).

\section{Conclusions}

We were able to objectively identify and discriminate between frailty categories using UEF parameters including speed of elbow flexion (slowness marker), strength of muscles in 
performing the task (weakness marker), exhaustion in performing elbow flexion, and flexibility of the elbow joint. It was possible to predict frailty with a sensitivity of $78 \%$ and a specificity of $82 \%$ when compared with the TSFI test. The UEF test is independent of walking assessments and it reflects several functional performance characteristics related to frailty, and also it is more practical to use in a hospital setting and bedbound patients. Although all UEF assessments were performed following fall events, we observed that UEF can predict frailty status (which was compared to the modified Rockwood assessment of conditions prior to the fall event), with a high accuracy. Further, within the current experimental setting we demonstrated that UEF may be representative of overall physical function status, as it was closely correlated with historical fall risk in older adults.

\section{COMPETING INTERESTS}

The authors declare that they have no competing interests.

\section{AUTHORS CONTRIBUTIONS}

NT: Concept and design, study coordination, analysis and interpretation of data, preparation of manuscript. BJ: Concept and design, clinical supervision, interpretation of data; MRH: Patient recruitment, study coordination, acquisition of data, and data analysis; TOJ: study coordination, acquisition of data; JM, Concept and design, interpretation of data; HAP: interpretation of data; BN: Concept and design, interpretation of data. All authors contributed in critically revising the manuscript and have given final approval of the version to be published.

\section{Acknowledgements}

We thank Bardiya Zangbar for data collection and clinical coordination. Partial support was provided by the National Institutes of Health/National Institute for Biomedical Imaging and Bioengineering (award number 1R25EB012973) and the Flinn Foundation (award number 
1907). The content is solely the responsibility of the authors and does not necessarily represent the official views of the Flinn Foundation or the National Institute for Biomedical Imaging and Bioengineering. 


\section{References}

1. Vincent GK, Velkoff VA. The next four decades: The older population in the United States: 2010 to 2050: US Department of Commerce, Economics and Statistics Administration, US Census Bureau; 2010.

2. 2010 APoOA. Administration on Aging, United States, Department of Health and Human Services.

http://wwwaoagov/aoaroot/aging statistics/profile/2010/docs/2010profilepdf. 2010.

3. Rowe JW, Kahn RL. Human aging: usual and successful. Science. 1987;237(4811):143-9.

4. Fried LP, Tangen CM, Walston J, et al. Frailty in older adults: Evidence for a phenotype. Journals Of Gerontology Series A-Biological Sciences And Medical Sciences. 2001 Mar;56(3):M146-M56.

5. Rockwood K, Andrew M, Mitnitski A. A comparison of two approaches to measuring frailty in elderly people. Journals Of Gerontology Series A-Biological Sciences And Medical Sciences. 2007 Jul;62(7):738-43.

6. Winograd CH, Gerety M, Chung M, Goldstein M, Dominguez Jr F, Vallone R. Screening for frailty: criteria and predictors of outcomes. Journal of the American Geriatrics Society. 1991;39(8):778-84.

7. Makary MA, Segev DL, Pronovost PJ, et al. Frailty as a predictor of surgical outcomes in older patients. Journal of the American College of Surgeons. 2010;210(6):901-8.

8. Toosizadeh N, Mohler J, Najafi B. Assessing Upper Extremity Motion: An Innovative Method to Identify Frailty. Journal of the American Geriatrics Society. 2015;63(6):1181-6.

9. Verghese J, Holtzer R, Lipton RB, Wang C. Quantitative gait markers and incident fall risk in older adults. The Journals of Gerontology Series A: Biological Sciences and Medical Sciences. 2009;64(8):896-901.

10. Van Kan GA, Rolland Y, Andrieu S, et al. Gait speed at usual pace as a predictor of adverse outcomes in community-dwelling older people an International Academy on Nutrition and Aging (IANA) Task Force. The journal of nutrition, health \& aging. 2009;13(10):881-9.

11. Association WM. World Medical Association Declaration of Helsinki: ethical principles for medical research involving human subjects. Jama. 2013;310(20):2191.

12. Joseph B, Pandit V, Zangbar B, et al. Validating trauma-specific frailty index for geriatric trauma patients: a prospective analysis. Journal of the American College of Surgeons. 2014;219(1):10-7. e1. 
13. Brown M, Sinacore DR, Binder EF, Kohrt WM. Physical and performance measures for the identification of mild to moderate frailty. The Journals of Gerontology Series A: Biological Sciences and Medical Sciences. 2000;55(6):M350-M5.

14. Lawrence I, Lin K. A concordance correlation coefficient to evaluate reproducibility. Biometrics. 1989:255-68.

15. Gill TM, Allore HG, Gahbauer EA, Murphy TE. Change in disability after hospitalization or restricted activity in older persons. JAMA. 2010;304(17):1919-28.

16. Fried LP, Tangen CM, Walston J, et al. Frailty in older adults evidence for a phenotype. The Journals of Gerontology Series A: Biological Sciences and Medical Sciences. 2001;56(3):M146-M57.

17. Fukagawa NK, Wolfson L, Judge J, Whipple R, King M. Strength is a major factor in balance, gait, and the occurrence of falls. The Journals of Gerontology Series A: Biological Sciences and Medical Sciences. 1995;50(Special Issue):64-7.

18. Wickham C, Cooper C, Margetts B, Barker D. Muscle strength, activity, housing and the risk of falls in elderly people. Age and ageing. 1989;18(1):47-51.

19. Baker SP, O'neill B. The injury severity score: an update. Journal of Trauma and Acute Care Surgery. 1976;16(11):882-5.

20. Mohler J, Najafi B, Fain M, Ramos KS. Precision Medicine: A Wider Definition. J Am Geriatr Soc. 2015 Sep;63(9):1971-2. 
Table 1: UEF parameter definitions.

\begin{tabular}{ll}
\hline Parameter & Definition \\
\hline Speed & Mean value of the elbow angular velocity range (maximum minus minimum speed) \\
\hline Flexibility & Mean value of the elbow flexion range \\
\hline Power & $\begin{array}{l}\text { Mean value of product of the angular acceleration range and the range of angular } \\
\text { velocity }\end{array}$ \\
\hline Rise time & Mean value of the time required to reach the maximum angular velocity \\
\hline Moment & $\begin{array}{l}\text { Mean value of the maximum moment on elbow within each flexion/extension; } \\
\text { estimated from the moment of inertia of the forearm and the hand, and elbow } \\
\text { motion }\end{array}$ \\
\hline Speed variability & $\begin{array}{l}\text { Coefficient of variation (standard deviation divided by the mean) of the angular } \\
\text { velocity range }\end{array}$ \\
\hline Speed reduction & $\begin{array}{l}\text { Difference in the angular velocity range between the last and the first five seconds } \\
\text { of elbow flexion as a percentage of the initial angular velocity range }\end{array}$ \\
\hline Flexion number & \begin{tabular}{l} 
Number of flexion/extensions during 20 seconds \\
\hline
\end{tabular}
\end{tabular}


Table 2: Demographic information and UEF parameters for frailty groups defined by TSFI. The asterisk symbol represents a significant between-group difference.

\begin{tabular}{|c|c|c|c|c|}
\hline Variable & Non-frail & Frail & $p$-value & Effect Size \\
\hline TSFI score (SD) & $0.15(0.08)$ & $0.44(0.16)$ & - & - \\
\hline Number, $\mathrm{n}$ (\% of total) & $52(51 \%)$ & $49(49 \%)$ & - & - \\
\hline Male, $\mathrm{n}$ ( $\%$ of the group) & $27(52 \%)$ & $21(43 \%)$ & 0.61 & - \\
\hline Age, year (SD) & $78(10)$ & $80(9)$ & 0.20 & 0.21 \\
\hline Stature, $\mathrm{cm}(\mathrm{SD})$ & $167.43(9.85)$ & $167.23(10.09)$ & 0.92 & 0.02 \\
\hline Body mass, kg (SD) & $71.37(15.98)$ & $69.90(15.40)$ & 0.64 & 0.09 \\
\hline Body mass index, $\mathrm{kg} / \mathrm{m}^{2}(\mathrm{SD})$ & $25.30(4.46)$ & $24.93(4.85)$ & 0.69 & 0.08 \\
\hline Number of falls $\dagger, n(S D)$ & $0.79(1.23)$ & $4.14(5.12)$ & $<.0001^{*}$ & 0.90 \\
\hline UEF Parameters & Non-frail & Frail & $p$-value $\ddagger$ & Effect Size \\
\hline Speed, deg/s (SD) & $838.83(281.67)$ & $458.24(220.56)$ & $<.0001^{*}$ & 1.50 \\
\hline Flexibility, deg (SD) & $101.95(25.93)$ & $74.45(30.47)$ & $<.0001^{*}$ & 0.97 \\
\hline Power, $\mathrm{deg}^{2} / \mathrm{s}^{3} \times 100000$ (SD) & $133.58(115.20)$ & $35.19(52.94)$ & $<.0001^{*}$ & 1.10 \\
\hline Rise time, s / 100 (SD) & $24.95(7.16)$ & $32.78(11.05)$ & $<.001^{*}$ & 0.73 \\
\hline Moment, Nm (SD) & $1.04(0.69)$ & $0.45(0.32)$ & $<.0001^{*}$ & 1.10 \\
\hline Speed variability, percentage (SD) & $31.86(36.82)$ & $34.52(54.17)$ & 0.84 & 0.06 \\
\hline Speed reduction, percentage (SD) & $7.76(9.73)$ & $13.34(12.00)$ & $<.01^{*}$ & 0.51 \\
\hline Number of flexions, $\mathrm{n}(\mathrm{SD})$ & $25.35(7.36)$ & $17.20(6.37)$ & $<.0001^{*}$ & 1.18 \\
\hline
\end{tabular}

$\uparrow$ Number of falls in a prior year before measurement

$\ddagger p$-value for between-group differences adjusted for age, gender, and body mass index (BMI) 
Table 3: Results of the multivariate regression model. Dependent variable: TSFI frailty score; independent variables: UEF parameters (with significant association with frailty from ANOVA), age, gender, and body mass index. The asterisk symbol represents a significant independent association.

\begin{tabular}{lcccccc}
\hline $\begin{array}{l}\text { Independent } \\
\text { Variables }\end{array}$ & $\begin{array}{c}\text { Parameter } \\
\text { Estimates }\end{array}$ & $\begin{array}{c}\text { Standard } \\
\text { Errors }\end{array}$ & $\boldsymbol{t}$ Ratio & $\boldsymbol{p}$-value & $\begin{array}{c}\mathbf{9 5 \%} \mathrm{Cl} \\
\text { (Lower) }\end{array}$ & $\begin{array}{c}\mathbf{9 5 \%} \mathbf{C l} \\
\text { (Upper) }\end{array}$ \\
\hline Speed, deg/s & $-8.09 \times 10^{-04}$ & $3.15 \times 10^{-04}$ & -2.57 & $0.01^{*}$ & $-14.38 \times 10^{-04}$ & $-1.80 \times 10^{-04}$ \\
\hline Flexibility, deg & $1.83 \times 10^{-03}$ & $1.69 \times 10^{-03}$ & 1.08 & 0.29 & $-1.56 \times 10^{-03}$ & $5.22 \times 10^{-03}$ \\
\hline Power, $\mathrm{deg}^{2} / \mathrm{sec}^{3}$ & $1.80 \times 10^{-08}$ & $0.69 \times 10^{-08}$ & 2.60 & $0.01^{*}$ & $0.41 \times 10^{-08}$ & $3.18 \times 10^{-08}$ \\
\hline Rise time, sec & $-1.80 \times 10^{-01}$ & $2.82 \times 10^{-01}$ & -0.64 & 0.53 & $-7.44 \times 10^{-01}$ & $3.84 \times 10^{-01}$ \\
\hline Moment, Nm & $-8.83 \times 10^{-02}$ & $9.24 \times 10^{-02}$ & -0.96 & 0.34 & $-27.31 \times 10^{-02}$ & $9.66 \times 10^{-02}$ \\
\hline Speed reduction, \% & $3.95 \times 10^{-03}$ & $1.58 \times 10^{-03}$ & 2.49 & $0.02^{*}$ & $0.78 \times 10^{-03}$ & $7.12 \times 10^{-03}$ \\
\hline Flexion Number, $\mathrm{n}$ & $-3.99 \times 10^{-03}$ & $5.44 \times 10^{-03}$ & -0.73 & 0.47 & $-14.86 \times 10^{-03}$ & $6.88 \times 10^{-03}$ \\
\hline Gender, [female] & $-1.89 \times 10^{-02}$ & $2.65 \times 10^{-02}$ & -0.71 & 0.48 & $-7.18 \times 10^{-02}$ & $3.41 \times 10^{-02}$ \\
\hline Age, year & $36.10 \times 10^{-03}$ & $2.06 \times 10^{-03}$ & 0.18 & 0.86 & $-3.76 \times 10^{-03}$ & $4.48 \times 10^{-03}$ \\
\hline BMl, kg/m 2 & $2.49 \times 10^{-03}$ & $4.62 \times 10^{-03}$ & 0.54 & 0.59 & $-6.75 \times 10^{-03}$ & $11.74 \times 10^{-03}$ \\
\hline
\end{tabular}


Figures Legends

Figure 1: Differences between UEF parameters between frailty groups defined by TSFI.

Significant differences are defined by asterisk symbols.

Figure 2: Association between UEF and TSFI scores within training and validation datasets. For the training dataset, demographic information and all UEF parameters with significant ANOVA association with the TSFI score were included. The UEF model was developed using parameters in the training dataset that showed independent association with the TSFI score

including speed, power, and speed reduction. Results for predicting the TSFI score using this model within the validation dataset is presented. 